\title{
Actividad hipoglucemiante in vitro de los polisacáridos digeridos de Nostoc sphaericum Vaucher ex Bornet \& Flahault (cushuro)
}

Miguel Angel Inocente Camones* 1; Bertha Jurado Teixeira 2; Eva Ramos Llica 2; Britt Alvarado Chávez 2; César Fuertes Ruiton 2; Luisa Cárdenas Montoya ${ }^{2}$; Betsabeth Rivera Castillo ${ }^{2}$

\section{RESUMEN}

Objetivo: Evaluar el potencial inhibitorio de las enzimas a-amilasa y a-glucosidasa, así como la inhibición de la difusión de la glucosa, por los polisacáridos aislados de Nostoc Sphaericum Vaucher ex Bornet \& Flahault tratados mediante digestión gastrointestinal simulada a nivel in vitro.

Materiales y métodos: Los polisacáridos obtenidos de microalga fueron purificados y tratados mediante digestibilidad gastrointestinal in vitro, con la finalidad de simular su digestión.

Se evaluó la actividad hipoglucemiante mediante los métodos de inhibición de la a-glucosidasa, a-amilasa y la difusión de la glucosa de los polisacáridos digeridos, comparados con el medicamento acarbosa.

Resultados: Los resultados mostraron que la concentración de $50 \mathrm{mg} / \mathrm{mL}$ de polisacáridos digeridos disminuye en un $85,26 \%$ la difusión de la glucosa a nivel in vitro. La concentración inhibitoria $\left(\mathrm{Cl}_{50}\right)$ de la enzima a-glucosidasa fue de $314,23 \pm 4,87 \mathrm{mg} / \mathrm{mL}$ y la enzima a-amilasa $113,59 \pm 3,10 \mathrm{mg} / \mathrm{mL}$.

Conclusiones: Los polisacáridos de Nostoc sphaericum tratados mediante digestión gastrointestinal in vitro, mostraron capacidad de inhibir las enzimas a-amilasa, a-glucosidasa y la difusión de glucosa; motivo por el cual la especie sería considerado promisoria para un posible tratamiento coadyuvante de la diabetes tipo 2 .

Palabras clave: Diabetes mellitus tipo 2; Alfa-amilasas; Alfa-glucosidasas; Glucosa (Fuente: DeCS BIREME).

\section{In vitro hypoglycemic activity of polysaccharides digested from Nostoc sphaericum Vaucher ex Bornet \& Flahault (cushuro)}

\section{ABSTRACT}

Objective: To evaluate the inhibitory potential of the a-amylase and a-glucosidase enzymes, as well as the inhibition of glucose diffusion, using polysaccharides isolated from Nostoc sphaericum Vaucher ex Bornet \& Flahault and treated by in vitro simulated gastrointestinal digestion.

Materials and methods: The polysaccharides obtained from the microalga were purified and treated through in vitro gastrointestinal digestibility in order to simulate digestion. The hypoglycemic activity was evaluated by methods such as the inhibition of a-glucosidase and a-amylase, and glucose diffusion of the digested polysaccharides, and compared with the medication acarbose.

Results: The results showed that the concentration of $50 \mathrm{mg} / \mathrm{mL}$ of digested polysaccharides decreases by $85.26 \%$ the spread of in vitro glucose. The inhibitory concentration $\left(\mathrm{IC}_{50}\right)$ of the a-glucosidase enzyme was $314.23 \pm 4.87$ $\mathrm{mg} / \mathrm{mL}$ and that of the a-amylase enzyme was $113.59 \pm 3.10 \mathrm{mg} / \mathrm{mL}$.

Conclusions: Nostoc sphaericum polysaccharides treated by in vitro gastrointestinal digestion showed the ability to inhibit the a-amylase and a-glucosidase enzymes, and glucose diffusion. Therefore, this species would be considered promising for a possible adjuvant treatment of type 2 diabetes.

Keywords: Type 2 diabetes mellitus; Alpha-amylases; Alpha-glucosidases; Glucose (Source: MeSH NLM).

1. Universidad de San Martín de Porres, Facultad de Medicina Humana, Centro de Investigación de Bioquímica y Nutrición. Lima, Perú.

2. Universidad Nacional Mayor de San Marcos, Facultad de Farmacia y Bioquímica. Lima, Perú.

* Autor corresponsal. 


\section{INTRODUCCIÓN}

La diabetes mellitus comprende un grupo de trastornos metabólicos frecuentes que comparten el fenotipo de la hiperglucemia. La diabetes tipo 2 es un grupo heterogéneo de trastornos que se caracterizan por grados variables de resistencia a la insulina, menor secreción de dicha hormona y una mayor producción de glucosa. La diabetes mellitus tipo 2 es precedida por un período de homeostasis anormal de la glucosa clasificado como intolerancia a la glucosa en ayuno o intolerancia a la glucosa (1). Por lo tanto, un enfoque terapéutico para el tratamiento de la diabetes es disminuir la hiperglucemia (2); esto se puede lograr por la inhibición de las enzimas que hidrolizan los hidratos de carbono como la a-glucosidasa y a-amilasa, así como la inhibición de la difusión de la glucosa en la digestión gastrointestinal (3). La a-amilasa está involucrada con la descomposición de los hidratos de carbono de cadena larga y la a-glucosidasa en la descomposición del almidón y disacáridos en glucosa. Los inhibidores de la a-amilasa y a-glucosidasa son el objetivo para el desarrollo de compuestos para el tratamiento de la diabetes. Los inhibidores de a-glucosidasa se utilizan como medicamentos orales para tratar la diabetes mellitus tipo 2, actúan como inhibidores competitivos de la enzima a-glucosidasa necesaria para digerir los carbohidratos ${ }^{(4-6)}$; sin embargo, los fármacos inhibidores de las enzimas utilizadas actualmente causan efectos secundarios gastrointestinales como la diarrea, flatulencia, distensión abdominal, etc ${ }^{(6)}$. Los inhibidores de a-glucosidasa y a-amilasa obtenidos de origen vegetal pueden ser utilizados como una terapia eficaz y alternativa para el tratamiento de la hiperglucemia postprandial, con efectos secundarios mínimos o nulos ${ }^{(7-9)}$. Los polisacáridos no amiláceos llamados fibras dietéticas solubles presentan una funcionalidad para reducir riesgos en enfermedades cardiometabólicas, debido a su viscosidad, excelente retención de agua para retrasar el vaciado gástrico y absorción de glucosa en el tracto gastrointestinal (10). La especie Nostoc sphaericum denominado "cushuro" es un tipo de microalga verde azulada que se encuentra ampliamente distribuida en lagunas, lagos, arroyos y zonas húmedas de la Región Puna del Perú, entre 3850 y 4500 m s.n. m. Esta microalga es consumida por los pobladores de la zona, en diferentes potajes y en su régimen dietético.

Diversos estudios han caracterizado e identificado polisacáridos en el género Nostoc ${ }^{(11-15)}$. Por ello, el objetivo del estudio fue evaluar el potencial inhibitorio de las enzimas a-amilasa y a-glucosidasa, así como la inhibición de la difusión de la glucosa, por los polisacáridos aislados de Nostoc sphaericum y tratados mediante digestión gastrointestinal simulada a nivel in vitro.

\section{MATERIALES Y MÉTODOS}

Se realizó un estudio analítico, experimental y longitudinal en el Centro de Investigación de Bioquímica y Nutrición (CIBN) de la Facultad de Medicina Humana de la Universidad de San Martín de Porres (FMH-USMP) y el Laboratorio de Farmacognosia de la Facultad de Farmacia y Bioquímica de la Universidad Nacional Mayor de San Marcos. El alga cianofícea Nostoc sphaericum fue colectada en la laguna Conococha en Chiquián (Huaraz) a 3400 m s. n. m. en el departamento de Ancash, Perú (Figura 1) y se depositó en el Museo de Historia Natural de la Universidad Nacional Mayor de San Marcos (Voucher USM 297250).

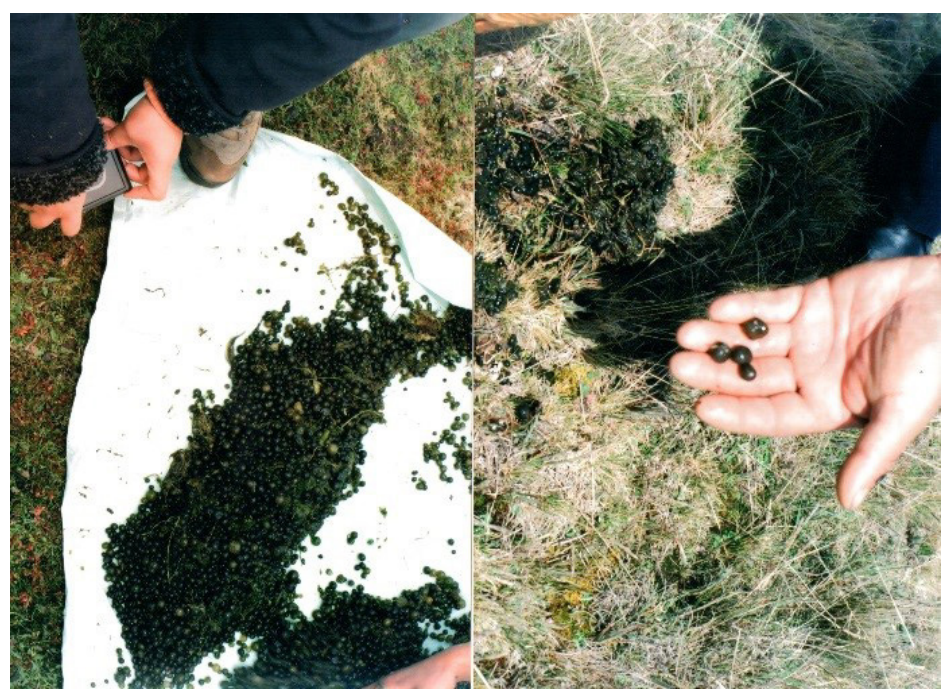

Figura 1. Recolección de Nostoc sphaericum en la laguna de Conococha, Huaraz, Perú 
La extracción y purificación de polisacáridos, se realizó según la metodología desarrollada por Wang et al. (11) y Jurado et al. ${ }^{(12)}$. Las muestras seleccionadas fueron almacenadas de 0 a $3{ }^{\circ} \mathrm{C}$ hasta su deshidratación parcial. Luego de la deshidratación, se descongelaron en baño maría de $30{ }^{\circ} \mathrm{C}$ y se licuaron con agua destilada para mejorar la ruptura de las capas de polisacáridos. Se sometió a calentamiento durante 30 minutos a $80^{\circ} \mathrm{C}$, se enfrío y filtró en filtro organdí lo que genera un líquido viscoso (fracción $A$ ) y un residuo (fracción $B$ ). La fracción A se concentró a la mitad de su volumen y se mezcló con etanol $96^{\circ}$ en proporción de 1:3 respectivamente, y se obtuvo un precipitado blanco en forma de flóculo, separado para su estabilización. Se repitió el proceso de extracción con la fracción $\mathrm{B}$. Los precipitados obtenidos se secaron a $40{ }^{\circ} \mathrm{C}$ por 24 horas y se purificaron con lavados continuos con etanol $96^{\circ}$, y se obtuvieron los polisacáridos del alga. El rendimiento de extracción fue de 16,47 gramos de polisacáridos por cada 100 gramos de alga.

Para el tratamiento de polisacáridos mediante digestibilidad in vitro, se utilizó la metodología de Fassler et al. ${ }^{(16)}$. La muestra consiste en polisacáridos obtenidos con un $5 \%$ de humedad. Para simular la digestión, se suspendieron 10 gramos de polisacáridos en $167 \mathrm{~mL}$ de buffer fosfato $20 \mathrm{mM}, \mathrm{pH} 6,9\left(\mathrm{Na}_{2} \mathrm{HPO}_{4} 1,42 \mathrm{~g} / \mathrm{L}, \mathrm{KH}_{2} \mathrm{PO}_{4}\right.$ $1,36 \mathrm{~g} / \mathrm{L}, \mathrm{NaCl} 0,58 \mathrm{~g} / \mathrm{L}$ ) y se procedió a incubarlos en una solución de a-amilasa salival humana (Sigma A1031$1 \mathrm{KU}, 10 \mathrm{mg} / \mathrm{mL}$, en $\mathrm{CaCl} 21 \mathrm{mM}$ ), durante 15 minutos. Para simular la digestión gástrica, se disminuyó hasta $\mathrm{pH} 2$ con $\mathrm{HCl} 0,5 \mathrm{~N}$, y se agregó en una suspensión de pepsina porcina (Sigma P7000-25G, $1 \mathrm{mg} / \mathrm{mL}$ en $\mathrm{NaCl}$ $9 \mathrm{~g} / \mathrm{L}$ ) durante 30 minutos. Para simular la digestión intestinal en la primera fracción del intestino delgado, se ajustó hasta $\mathrm{pH}$ 6,9 con $\mathrm{NaOH}$ y se agregó pancreatina porcina (Sigma P7545-25G, 0,5 mg/mL, en $\mathrm{CaCl} 225$ $\mathrm{mM}$ ) durante 3 horas en presencia de bilis bovina (Sigma B8381-10G), a $37{ }^{\circ} \mathrm{C}$. El producto de la digestión fue separado y congelado a $0{ }^{\circ} \mathrm{C}$ para las pruebas posteriores. Previamente, se comprobó la actividad enzimática de las enzimas participantes de la digestibilidad.

Ensayo de inhibición in vitro de la difusión de la glucosa

El método fue adaptado de Khaleel et al. (17). Luego del tratamiento de los polisacáridos en el modelo de simulación de la digestión gastrointestinal, se mezclaron $1 \mathrm{~mL}$ de las concentraciones de 0,$5 ; 1,0 ; 5,0 ; 10,0 ; 25,0$ y $50,0 \mathrm{mg} / \mathrm{mL}$ de polisacáridos con $2 \mathrm{~mL}$ de una solución de glucosa 0,22 $\mathrm{M}$ y cloruro de sodio 0,15 $\mathrm{M}$, en tubos de membrana de diálisis $(1 \mathrm{kD})$. El tubo se selló por ambos lados y se colocó en un beaker con $45 \mathrm{~mL}$ de cloruro de sodio $0,15 \mathrm{M}$, en un agitador orbital ( $150 \mathrm{RPM})$ a $25^{\circ} \mathrm{C}$ y se midió la concentración de la glucosa con un kit de glucosa oxidasa en 1, 3, 5, 24 y 27 horas.
Ensayo de inhibición in vitro de la enzima a-glucosidasa

La detección de la inhibición del a-glucosidasa se realizó mediante la técnica propuesta por Matsui et al. (18). Una unidad de a-glucosidasa libera $1 \mu \mathrm{mol}$ de $D$-glucosa o p-nitrofenil (PNP) a partir de p-nitrofenil a-Dglucopiranósido (PNP-G) (Sigma N1377-1G) por minuto a pH 6,8 y $37^{\circ} \mathrm{C}$. La solución enzimática fue preparada con 5,7 $U$ de a-glucosidasa (Sigma G5003-100U) en un volumen de $3,22 \mathrm{~mL}$ de agua desionizada fría. Para realizar la reacción se utilizaron tubos de $5 \mathrm{~mL}$ en el cual se adiciona $35 \mu \mathrm{L}$ de solución enzimática se mezcló con los polisacáridos digeridos a varias concentraciones (25, $50,75,150,250$ y $500 \mathrm{mg} / \mathrm{mL}$ ) y se mantuvo a $37^{\circ} \mathrm{C}$ por 5 minutos, en baño maría para alcanzar el equilibrio. La reacción se inició al agregar $930 \mu \mathrm{L}$ de PNP-G $(0,914$ $\mathrm{mM}$ en $2500 \mu \mathrm{L}$ de tampón fosfato salino $67 \mathrm{mM}(\mathrm{NaCl}$ $81 \%, \mathrm{KCl} 2 \%, \mathrm{Na}_{2} \mathrm{PO}_{4} 15 \%, \mathrm{KH}_{2} \mathrm{PO}_{4} 2 \%$ ) y toda la mezcla fue incubada a $37^{\circ} \mathrm{C}$ durante 15 minutos. Transcurrido el tiempo de incubación se adicionó a cada tubo $1 \mathrm{~mL}$ de solución TRIS $(0,5 \mathrm{M})$ para detener completamente la reacción, y se obtiene un color amarillo y un volumen final $2 \mathrm{~mL}$. Luego se mezcló por inversión y se realizó la lectura en espectrofotómetro UV-VIS a $400 \mathrm{~nm}$.

\section{Ensayo de inhibición in vitro de la enzima a-amilasa}

La detección de inhibición de a amilasa se realizó mediante la técnica propuesta por Tsujita et al. ${ }^{(19)}$. Una unidad de a-amilasa libera $1 \mathrm{mg}$ de maltosa en 3 minutos a $\mathrm{pH} 6,9\left(20^{\circ} \mathrm{C}\right)$. La solución enzimática fue preparada con 39,56 U (Sigma A3176-1MU) en $20 \mathrm{~mL}$ de agua desionizada fría. Se llevó a ebullición $125 \mu \mathrm{L}$ de almidón $1 \%$ en $20 \mathrm{mM}$ de buffer fosfato $\left(\mathrm{NaCl} 81 \%, \mathrm{KCl} 2 \%, \mathrm{Na}_{2} \mathrm{PO}_{4} 15 \%, \mathrm{KH}_{2} \mathrm{PO}_{4}\right.$ $2 \%)$ por 15 minutos. Luego se mezcló con $125 \mu \mathrm{L}$ del polisacárido digerido a varias concentraciones $(10,50$, 100,500 y $1000 \mu \mathrm{g} / \mathrm{mL}$ ) a $20^{\circ} \mathrm{C}$ por 5 minutos. Se agregó $125 \mu \mathrm{L}$ de la solución enzimática y se incubó a $20^{\circ} \mathrm{C}$ por 3 minutos. La reacción fue detenida por adición de $125 \mu \mathrm{L}$ de una solución DNS ( $96 \mathrm{mM}$ de ácido 3,5-dinitrosalicílico (Sigma D0550-10G)) y tartrato de sodio y potasio en $\mathrm{NaOH}$ $2 \mathrm{M})$. Luego la muestra se llevó a un baño maría a $80^{\circ} \mathrm{C}$ por 15 minutos. Finalmente, la solución fue enfriada a 0 ${ }^{\circ} \mathrm{C}$ por 5 minutos y se agregó $2 \mathrm{~mL}$ de agua desionizada, con lo que se consigue un color anaranjado rojizo y un volumen final de $2,5 \mathrm{~mL}$, se mezcló por inversión y se realizó la lectura de las absorbancias a $540 \mathrm{~nm}$.

Cálculos del porcentaje de inhibición de las enzimas a-glucosidasa y a-amilasa

El porcentaje de inhibición para las enzimas fue expresado mediante la siguiente ecuación:

$$
\% \text { Inhibición }=(1-[(B-D) /(A-C]) \times 100
$$


Donde B fue la absorbancia de la muestra, D la absorbancia del blanco, A la absorbancia del control 1 (sin polisacárido) y C la absorbancia del control 2 (sin polisacárido y sin solución enzimática). $\mathrm{La} \mathrm{Cl}_{50}$ se define como la concentración de a-glucosidasa o a-amilasa requerida para inhibir el $50 \%$ de la actividad de estas, bajo las condiciones del ensayo.

\section{Análisis estadístico}

Los ensayos se presentan como \% Inhibición \pm DS. Se realizó la comparación estadística entre grupos mediante el análisis de varianza (ANOVA) y la prueba de Tukey para el análisis post hoc con $p<0,05$, mediante el software SPSS 21.

\section{RESULTADOS}

Ensayo de inhibición in vitro de la difusión de la glucosa

En la tabla 1 se observa que los niveles de glucosa en la solución externa del tubo de membrana de diálisis disminuyen gradualmente según la concentración de polisacáridos digeridos presentes en la solución. Se obtuvo como $\mathrm{Cl}_{50}$ el valor de 7,44 $\pm 0,61 \mathrm{mg} / \mathrm{mL}$.

Tabla 1. Efecto de los polisacáridos digeridos en la difusión de glucosa a través de una membrana de diálisis

\begin{tabular}{|c|c|c|c|c|c|c|}
\hline \multicolumn{7}{|c|}{ Concentración de glucosa en el tiempo (mg/mL - HORA) } \\
\hline $\begin{array}{l}\text { Muestra } \\
(\mathrm{mg} / \mathrm{mL})\end{array}$ & 1 & 3 & 5 & 24 & 27 & $\begin{array}{c}(\%) \\
\text { Inhibición }\end{array}$ \\
\hline Control & $67,40 \pm 0,92$ & $81,57 \pm 2,81$ & $98,60 \pm 3,20$ & $166,57 \pm 3,74$ & $170,7 \pm 2,17$ & ---- \\
\hline 0,5 & $56,20 \pm 2,61$ & $68,00 \pm 4,75$ & $83,47 \pm 4,85$ & $112,17 \pm 3,91$ & $118,8 \pm 6,31$ & 30,41 \\
\hline 1,0 & $47,20 \pm 3,15$ & $53,17 \pm 4,30$ & $71,80 \pm 2,07$ & $99,13 \pm 4,71$ & $102,50 \pm 3,82$ & 39,94 \\
\hline 5,0 & $47,60 \pm 1,32$ & $52,30 \pm 0,46$ & $62,23 \pm 1,68$ & $82,30 \pm 2,16$ & $86,70 \pm 2,94$ & 49,18 \\
\hline 10,0 & $32,97 \pm 0,38$ & $36,10 \pm 2,43$ & $43,40 \pm 1,14$ & $49,93 \pm 1,52$ & $54,30 \pm 3,57$ & 68,16 \\
\hline 25,0 & $26,80 \pm 4,14$ & $33,83 \pm 3,51$ & $35,53 \pm 2,18$ & $42,37 \pm 1,40$ & $47,40 \pm 2,46$ & 72,24 \\
\hline 50,0 & $11,37 \pm 1,16$ & $13,80 \pm 1,15$ & $17,07 \pm 1,57$ & $22,63 \pm 2,40$ & $25,20 \pm 3,14$ & 85,26 \\
\hline
\end{tabular}

${ }^{*} \mathrm{IC} 50=7,44 \pm 0,61 \mathrm{mg} / \mathrm{mL}$

Ensayo de inhibición in vitro de la enzima a-glucosidasa y a-amilasa

Los resultados de la evaluación de la actividad inhibitoria de las enzimas a-glucosidasa y a-amilasa se resumen en la tabla 2 y 3 , respectivamente. Los polisacáridos aislados y tratados en la digestión gastrointestinal mostraron una inhibición dependiente de la concentración de la enzima, con un $\mathrm{Cl} 50$ de $314,23 \mathrm{mg} / \mathrm{mL}$ para inhibir el a-glucosidasa y de $113,59 \mathrm{mg} / \mathrm{mL}$ para inhibir el a-amilasa.

Tabla 2. Efecto de los polisacáridos digeridos en la inhibición de la enzima a-glucosidasa

\begin{tabular}{cccc}
$\begin{array}{c}\text { Muestra } \\
(\mathbf{m g} / \mathbf{m L})\end{array}$ & $\begin{array}{c}\text { \% Inhibición de la } \\
\text { enzima }\end{array}$ & $\begin{array}{c}\text { Control } \\
\text { (GLUCOBAY, } \boldsymbol{\mu g} / \mathbf{m L})\end{array}$ & $\begin{array}{c}\text { \% Inhibición de } \\
\text { la enzima }\end{array}$ \\
\hline 25 & $4,83 \pm 0,75$ & $\mathbf{1 0}$ & $2,07 \pm 0,65$ \\
50 & $10,65 \pm 0,88$ & $\mathbf{5 0}$ & $10,46 \pm 0,00$ \\
75 & $16,44 \pm 0,91$ & $\mathbf{1 0 0}$ & $16,67 \pm 12,59$ \\
150 & $25,17 \pm 1,13$ & $\mathbf{5 0 0}$ & $30,80 \pm 16,63$ \\
250 & $52,03 \pm 0,81$ & $\mathbf{1 0 0 0}$ & $41,92 \pm 15,20$ \\
500 & $72,45 \pm 0,86$ & $\mathbf{2 0 0 0}$ & $59,58 \pm 17,65$ \\
& $\mathbf{C l}_{\mathbf{5 0}}(\mathbf{m g} / \mathbf{m L}) \mathbf{3 1 4 , 2 3} \mathbf{4 , 8 7}$ & $\mathbf{C l}_{\mathbf{5 0}}(\boldsymbol{\mu} \mathbf{g} / \mathbf{m L}) \mathbf{1 1 7 8 , 2 4} \pm \mathbf{1 8 , 4 5}$
\end{tabular}


Tabla 3. Efecto de los polisacáridos digeridos en la inhibición de la enzima a-amilasa

\begin{tabular}{|cc|}
\hline Muestra $(\mathbf{m g} / \mathbf{m L})$ & \% Inhibición de la enzima $\mathbf{P}$ \\
\hline $\mathbf{2 5}$ & $23,34 \pm 1,21$ \\
$\mathbf{5 0}$ & $28,90 \pm 3,01$ \\
$\mathbf{7 5}$ & $37,66 \pm 1,03$ \\
$\mathbf{1 0 0}$ & $47,91 \pm 1,05$ \\
$\mathbf{1 5 0}$ & $63, \mathbf{2 8} \pm 2,04$ \\
$\mathbf{2 5 0}$ & $89, \mathbf{4 3} \pm 0,63$ \\
& IC $\mathbf{5 0}=\mathbf{1 1 3 , 5 9} \pm \mathbf{3 , 1 0} \mathbf{~} \mathbf{m g} / \mathbf{m L}$ \\
\hline
\end{tabular}

\section{DISCUSIÓN}

Diversos estudios relacionan la acción positiva de los polisacáridos, mucílagos y gomas sobre la inhibición de la difusión de la glucosa e inhibición de la a-glucosidasa (20-26). Los mecanismos por los cuales las plantas pueden disminuir la glucosa implican la viscosidad de diferentes fibras o polisacáridos para obstaculizar la difusión de la glucosa y el enlentecimiento de la absorción y digestión de hidratos de carbono. En este trabajo se utilizaron polisacáridos de Nostoc sphaericum para investigar el mecanismo de reducción de la glucosa in vitro. Los resultados muestran que disminuyen la difusión de la glucosa; sin embargo, el modelo de difusión no puede reflejar el movimiento real del intestino. Por otra parte, el período del tiempo de estudio ( 27 horas) considerado por el modelo de Khaleel et al. (17), no es consistente con el momento de la absorción de la glucosa en la mucosa intestinal. Los resultados pueden justificarse por diversas hipótesis. Una de ellas, es que los polisacáridos aumentan la viscosidad y obstaculizan la difusión de glucosa; otra es que disminuyen el transporte de glucosa a través de la membrana. Aunque los mecanismos exactos por los que el polisacárido ejerce su actividad hipoglucemiante se desconocen. Sin embargo, su capacidad para retardar la absorción sin duda tiene una influencia importante en el metabolismo de los hidratos de carbono. Al evaluar la actividad inhibitoria de las enzimas, el polisacárido aislado y tratado en el modelo de digestión parece ser menos potente para inhibir a la a-glucosidasa en comparación de la acarbosa. Esto puede deberse a que la enzima es más sensible al fármaco purificado y que el polisacárido tiene una posible acción de aglomeración para retardar la acción de la enzima.

En conclusión, el presente trabajo demuestra la capacidad del polisacárido de Nostoc sphaericum para inhibir la difusión de la glucosa, la a-amilasa intestinal y a-glucosidasa al utilizar modelos in vitro. Por lo tanto, los polisacáridos de Nostoc sphaericum representan un potencial suplemento de la dieta en pacientes diabéticos tipo 2. Se requieren más estudios para aclarar si los efectos in vitro representan un potencial terapéutico a nivel de ensayo clínico.

Los polisacáridos de Nostoc sphaericum tratados mediante digestión gastrointestinal in vitro, mostraron capacidad de inhibir las enzimas a-amilasa, a-glucosidasa y la difusión de glucosa; motivo por el cual la especie sería considerada promisoria para un posible tratamiento coadyuvante de la diabetes tipo 2; sin embargo, se requiere los estudios preclínicos in vivo y clínicos para validar su uso medicinal.

\section{REFERENCIAS BIBLIOGRÁFICAS}

1. Kasper D, Fauci A, Hauser S, Longo D, Jameson L, Loscalzo J. Harrison Principios de Medicina Interna, $19^{\circ}$ ed. Barcelona: Editorial McGraw-Hill Interamericana; 2016.

2. Breuer HW. The postprandial blood glucose level - A new target for optimizing treatment of diabetes mellitus. Eur Heart J. 2000; D (2): D36-D38

3. Bhandari MR, Jong AN, Hong G, Kawabata J. A-Glucosidase and a-amylase inhibitory activities of Nepalese medicinal herb Parkhanbhed (Bergenia ciliata, Haw). Food .Food Chem. 2008; 106(1): 247-52.

4. Avellaneda I. Evaluación de la actividad inhibitoria de la a-glucosidasa (a-GLC) in vitro por extractos vegetales. [Tesis para optar al título de Químico Industrial]. Pereira: Universidad Tecnológica de Pereira; 2013: 51-66.

5. Sevilla O, Dubián O, Gómez L, López L. Actividad inhibitoria sobre a-glucosidasa y a-amilasa de extractos acuosos de algunas especias utilizadas en la cocina mexicana. CienciaUAT. 2013; 26(2): 42-47.

6. Cheng A, Fantus I. Oral antihyperglycemic therapy for type 2 diabetes mellitus. CMAJ. 2005; 172(2): 213-26.

7. Alejandro M, Jaramillo X, Ojeda S, Malagón O, Ramírez J. Actividad antioxidante y antihiperglucemiante de la especie medicinal Oreocallis grandiflora (Lam.) $\mathrm{R} \mathrm{Br}$, al sur del Ecuador. BLACPMA. 2013; 12(1): 59-68.

8. Kolawole $\mathrm{O}$, Akanji M. Inhibitory effect of leaf extract of Newbouldia laevis on the metabolic activities of a-glucosidase 
and a-amylase. Bangladesh J Pharmacol. 2013; 8(4): 371-377.

9. Edwards CA, Blackburn NA, Craigen L, Davison P, Tomlin J, Sudgen $\mathrm{K}$, et al. Viscosity of food gums determined in vitro related to their hypoglycemic actions. Am J Clin Nutr. 1987; 46(1): 72-77.

10. Jenkins AL, Jenkins DJ, Wolever T, Rogovik A, Jovanovski E, Bozikov V, et al. Comparable postprandial glucose reductions with viscous fiber blend enriched biscuits in healthy subjects and patients with diabetes mellitus: acute randomized controlled clinical trial. Croat Med J. 2008; 49(6): 772-82.

11. Wang HB, Wu SJ, Liu D. Preparation of polysaccharides from cianobacteria Nostoc commune and their antioxidant activities. Carbohydr Polym. 2014; 99: 553-55.

12. Jurado B, Fuertes C, Tomas G, Ramos E, Arroyo J, Cáceres J, et al. Estudio fisicoquímico, microbiológico y toxicológico de los polisacáridos del Nostoc commune y Nostoc sphaericum. Rev Per Quím Ing Quím. 2014; 17(1): 15-22.

13. Guo M, Ding G, Guo S, Li Z, Zhao L, Li K, et al. Isolation and antitumor efficacy evaluation of a polysaccharide from Nostoc commune Vauch. Food Funct. 2015; 6(9): 35-3044.

14. Diao Y, Han H, Li Y, Zhou J, Yang Z. [Internet]. Extraction, infrared spectral analysis and the antimicrobial activity on polysaccharide within Nostoc commune Vauch. 2nd International Conference on Environment, Energy and biotechnology. [internet]. 2013 [citado 1 de noviembre de 2018];51(12):59-63. Disponible en: http://www.ipcbee.com/ vol51/012-ICEEB2013-A10007.pdf

15. Volk RB, Venske K, Blaschek W. Structural investigation of a polysaccharide released by the cyanobacterium Nostoc insulare. J Appl Phycol. 2007; 19: 255-62.

16. Fassler C, Arrigoni E, Venema K, Hafner V, Brouns F, Amado R. Digestibility of resistant starch containing preparations using two in vitro models. Eur J Nutr. 2006; 45(8): 445-53.

17. Khaleel S, Sugantha V. In vitro antidiabetic activity of Psidium guajava leaves extracts. Asian Pac J Trop Dis. 2012; 2(1): S98-S100.

18. Matsui T, Yoshimoto C, Osajima K, Osajima Y. In vitro survey of alpha-glucosidase inhibitory food components. Biosci Biotechnol Biochem. 1996; 60(12): 2019-22.

19. Tsujita T, Takeshi T. US Patente $N^{\circ} 2006 / 030567$. US Patent application publication. Washington DC; 2006.

20. Bisht S, Kant R, Kumar V. a-D-Glucosidase inhibitory activity of polysaccharide isolated from Acacia tortilis gum exudate. Int J Biol Macromol. 2013; 59: 214-20.

21. Hsu WK, Hsu TH, Lin FY, Cheng YK, Yanq JP. Separation, purification, and a-glucosidase inhibition of polysaccharides from Coriolus versicolor LH1 mycelia. Carbohydr Polym. 2013; 92(1): 297-306.

22. Zhang L, Zhang N, Li J, Wang Z. New a-Glucosidase

Inhibitory Polysaccharides Isolated from Marine Green Algae Enteromorpha Linza. Adv Mat Res. 2013; 634-638: 1010-15.

23. Guocong L. Chemical compositions, a-glucosidase and a-amylase inhibitory activities of crude polysaccharides from the endodermis of shaddock (Citrus maxima). Arch Biol Sci. 2012; 64(1): 71-76.

24. Song $\mathrm{Y}$, Zhang $\mathrm{Y}$, Zhou T, Zhang $\mathrm{H}$, Hu X, Quanhong L. A preliminary study of monosaccharide composition and a-glucosidase inhibitory effect of polysaccharides from pumpkin (Cucurbita moschata) fruit. Int J Food Sci Technol. 2012; 47 (2): 357-61.

25. Palanuvej Ch, Hokputsa S. Tunsaringkarn T, Ruangrungsi N. In vitro glucose entrapment and alpha-glucosidase inhibition of mucilaginous substances from selected thai medicinal plants. Sci Pharm. 2009; 77(4): 837-49.

26. Pramitha V, Lipton A, Thangaraj M. Evaluation of $a$-and $B$-glucosidase inhibitory properties of macro-algae using intestinal extracts of marine snail, Thais rudolphi (Lamarck, 1822). Ind J Biotechnol. 2008; 7(1): 61-65.

Fuentes de financiamiento:

Este artículo ha sido financiado por los autores.

Conflictos de interés:

Los autores declaran no tener ningún conflicto de interés.

Correspondencia:

Miguel Ángel Inocente Camones

Dirección: Av. Alameda del Corregidor 1531, La Molina. Lima, Perú Teléfono: 3652300 anexo 148

Correo electrónico: minocentec@usmp.pe

Recibido: 05 de julio de 2018

Evaluado: 15 de agosto de 2018

Aprobado: 17 de setiembre de 2018 .

( C La revista. Publicado por Universidad de San Martín de Porres, Perú. (c) Br $^{\text {B }}$ Licencia de Creative Commons Artículo en acceso abierto bajo términos de Licencia Creative Commons Atribución 4.0 Internacional. (http://creativecommons.org/licenses/by/4.0/)

\section{ORCID iDs}

Miguel Angel Inocente Camones Bertha Jurado Teixeira Eva Ramos Llica Britt Alvarado Chávez César Fuertes Ruiton Luisa Cárdenas Montoya Betsabeth Rivera Castillo https://orcid.org/0000-0003-0397-4356 https://orcid.org/0000-0001-5988-7433 https://orcid.org/0000-0001-8594-5537 https://orcid.org/0000-0002-0601-6973 https://orcid.org/0000-0002-6170-3549 https://orcid.org/0000-0003-1388-9155 https://orcid.org/0000-0002-8349-6093 